\title{
Dextran-coated Gold Nanoparticles for the Assessment of Antimicrobial \\ Susceptibility
}

Sudip Nath, Charalambos Kaittanis, Alisa Tinkham and J. Manuel Perez ${ }^{*}$

\section{Supporting Information}

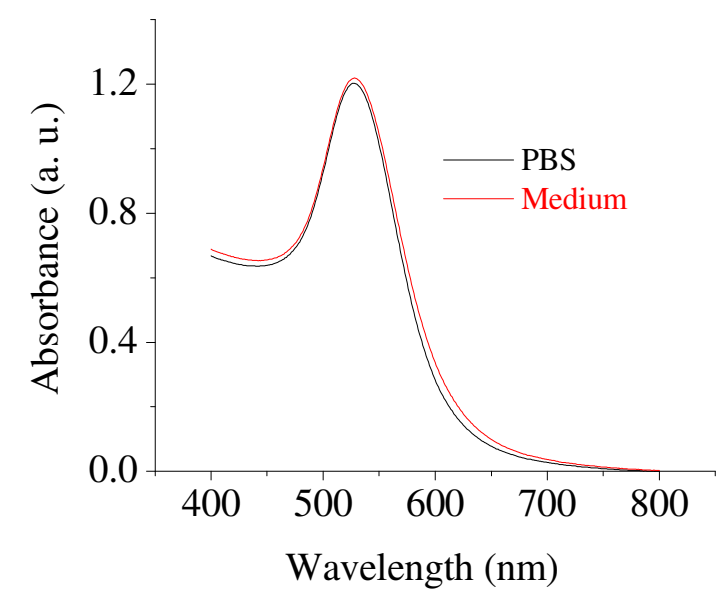

Fig. 1: UV-Visible spectra of Dex-Au-NP in non-assembled conditions.

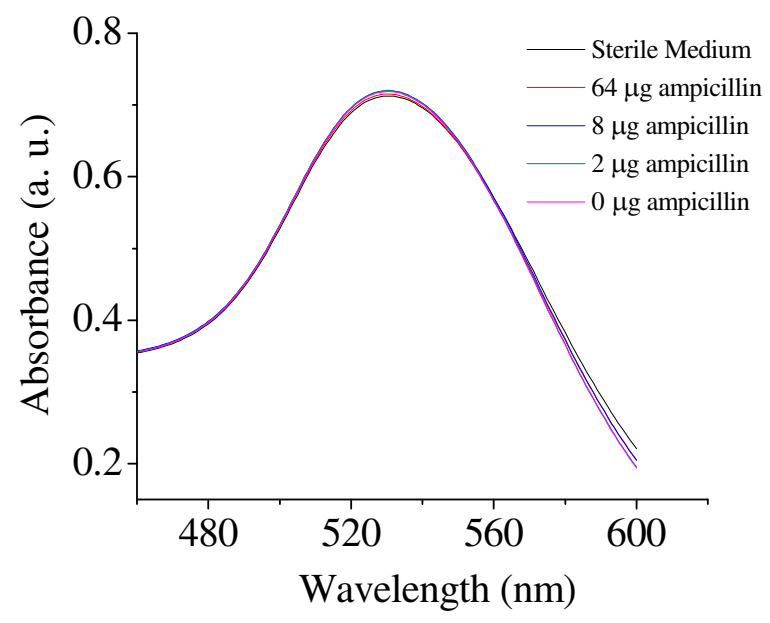

Fig. 2: In the absence of Con A, the Dex-Au-NPs were in a non-assembled state, exhibiting the same spectral characteristics, regardless of the presence of bacteria and antibiotic. The samples with ampicillin $(64,8$, and $2 \mu \mathrm{g})$ and the bacterial growth control ( $0 \mu \mathrm{g}$ ampicillin) contained aliquots from liquid cultures initially inoculated with $10^{6}$ CFU E. coli. 